\title{
A cascade reaction network mimicking the basic functional steps of adaptive immune response
}

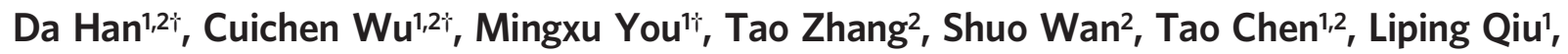 \\ Zheng Zheng ${ }^{2}$, Hao Liang ${ }^{1}$ and Weihong Tan ${ }^{1,2 \star}$
}

\begin{abstract}
Biological systems use complex 'information-processing cores' composed of molecular networks to coordinate their external environment and internal states. An example of this is the acquired, or adaptive, immune system (AIS), which is composed of both humoral and cell-mediated components. Here we report the step-by-step construction of a prototype mimic of the AIS that we call an adaptive immune response simulator (AIRS). DNA and enzymes are used as simple artificial analogues of the components of the AIS to create a system that responds to specific molecular stimuli in vitro. We show that this network of reactions can function in a manner that is superficially similar to the most basic responses of the vertebrate AIS, including reaction sequences that mimic both humoral and cellular responses. As such, AIRS provides guidelines for the design and engineering of artificial reaction networks and molecular devices.
\end{abstract}

A 11 organisms, including plants, have developed mechanisms of defence against pathogenic and other challenges, and thus solve a key biological problem: survival. A stunning example of this in humans is the development of learned, or adaptive, immunity, which is composed of both humoral and cellmediated components. Adaptive immunity involves the production of antibodies and immunological memory, but also cell-mediated responses, including the key steps of antigen presentation, antigen binding, co-stimulation and destruction of the foreign body (phagocytosis). More broadly, specialized cells, tissues and organs combine together as a network to recognize, respond and remember specific antigens or pathogens. Functionally, each step in the sequence of humoral and cellular immune response involves the activation and deployment of B and T lymphocytes via highly complex signalling pathways. A fully implemented artificial adaptive immune system (AIS) has not been well studied, possibly as a result of the difficulty in controlling programmability and transferring the high-level biological codes into simple artificial modules and physical implementations. In addition, the formation of robust molecular structures and the precise control of their temporal dynamics may pose other difficulties. However, in the present work, we hypothesize the feasibility of designing a network of reactions able to function in a manner similar to the vertebrate AIS, using DNA and enzymes as simplified artificial analogues to mimic the activation and deployment of an adaptive immune response at its most fundamental level.

This study reports the step-by-step construction of such a prototype, termed hereinafter as an adaptive immune response simulator, or AIRS. We first asked what building materials would best replicate the intricate networks of chemical reactions, signalling pathways and cell-cell communication that control host immunity ${ }^{1}$. As a carrier of genetic information with well-regulated and predictable structures, DNA offers an excellent platform for the design of reaction networks with arbitrary topologies and high programmability ${ }^{2,3}$. Building on the richness of DNA computing ${ }^{4,5}$ and DNA-enzyme manipulation ${ }^{6,7}$, synthetic DNA-based systems have been used to explore the possibilities of mimicking both simple and complex systems. For example, small-scale in vitro circuits that encode elementary functions, such as cascades ${ }^{8}$, bistable memory ${ }^{9,10}$ or oscillations ${ }^{11}$, have been engineered successfully. In addition, larger networks, including those that mimic neural networks $^{12}$ or predator-prey ecosystems ${ }^{13,14}$, confirm the value of DNA chemistry for implementing the type of system proposed in this study.

Pathogenic challenge results in the production of antibodies that lock onto a part of the foreign body known as an antigen. As this event triggers a complex cascade of cell-mediated reactions, we next asked which bioengineering techniques would best replicate the intricate networks of chemical reactions, signalling pathways and cell-cell communication that control host immunity. To address both questions, in a stepwise manner we report the construction of a chemical reaction network, AIRS, that couples DNA-enzyme cascade interactions, as described above, with DNA-strand-displacement cascades in which a new product can be activated by the presence of specific initiators ${ }^{15,16}$, and thus allows many such reactions to be linked into a cascade and even to form a complex network, in particular one such as adaptive immunity in which biological events follow each other sequentially in a rolling fashion.

\section{Results and discussion}

System construction. Once a foreign target has crossed the threshold of immune tolerance, the humoral and cellular components of the vertebrate immune system are called to action. A key function is antigen presentation, which provides the mechanism for recognition and response. B cells secrete antibodies, which bind to and tag an antigen, whereas $\mathrm{T}$ cells perform the job of attacking target cells. Both $\mathrm{T}$ and $\mathrm{B}$ cells also have roles in forming memory cells. As shown in Fig. 1, we divided this series of events into three steps (recognition and

'Molecular Science and Biomedicine Laboratory, State Key Laboratory of Chemo/Biosensing and Chemometrics, College of Chemistry and Chemical Engineering, College of Biology, Collaborative Innovation Center for Molecular Engineering for Theranostics, Hunan University, Changsha 410082, China. ${ }^{2}$ Center for Research at Bio/nano Interface, Department of Chemistry and Department of Physiology and Functional Genomics, Health Cancer Center, UF Genetics Institute and McKnight Brain Institute, University of Florida, Gainesville, Florida 32611-7200, USA. †These authors contributed equally to this work. *e-mail: tan@chem.ufl.edu 
Step 1: Recognition and tolerance
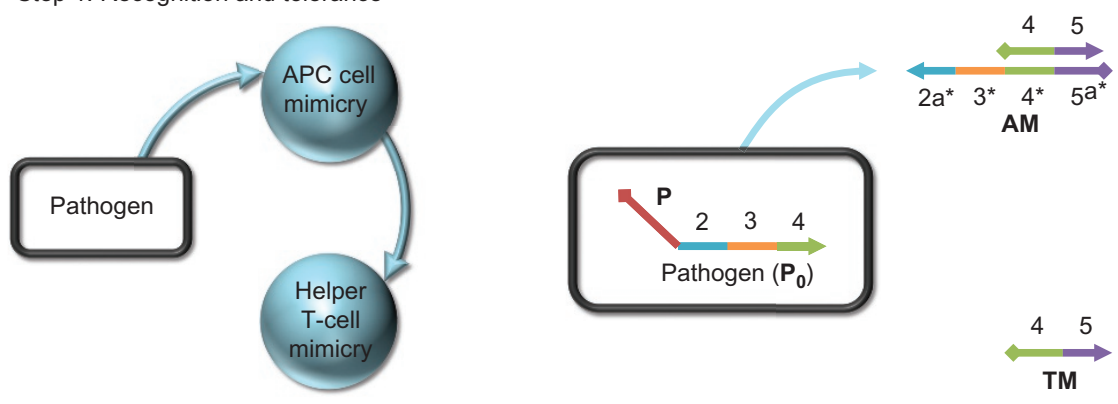

Step 2: Immune response
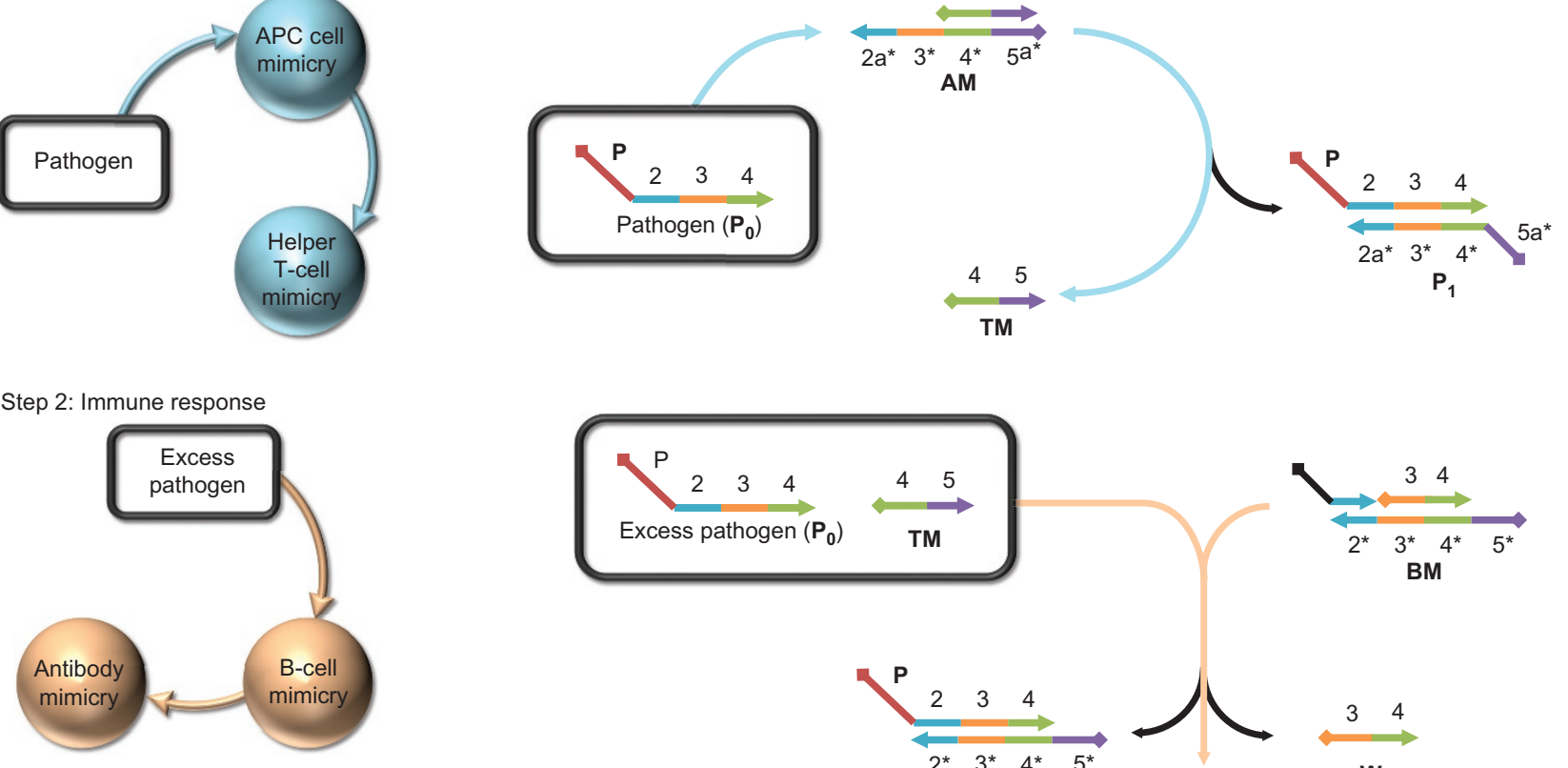$$
\text { 西 }
$$
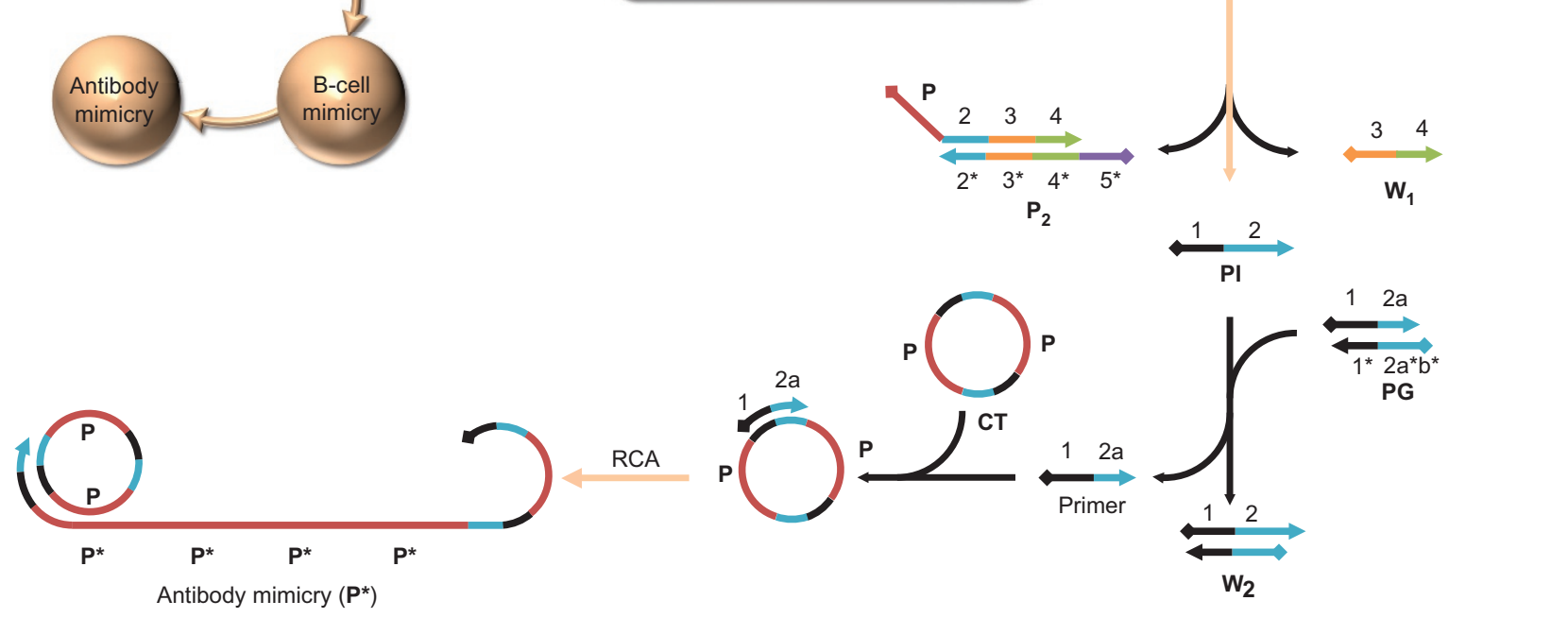

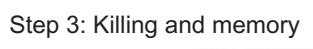

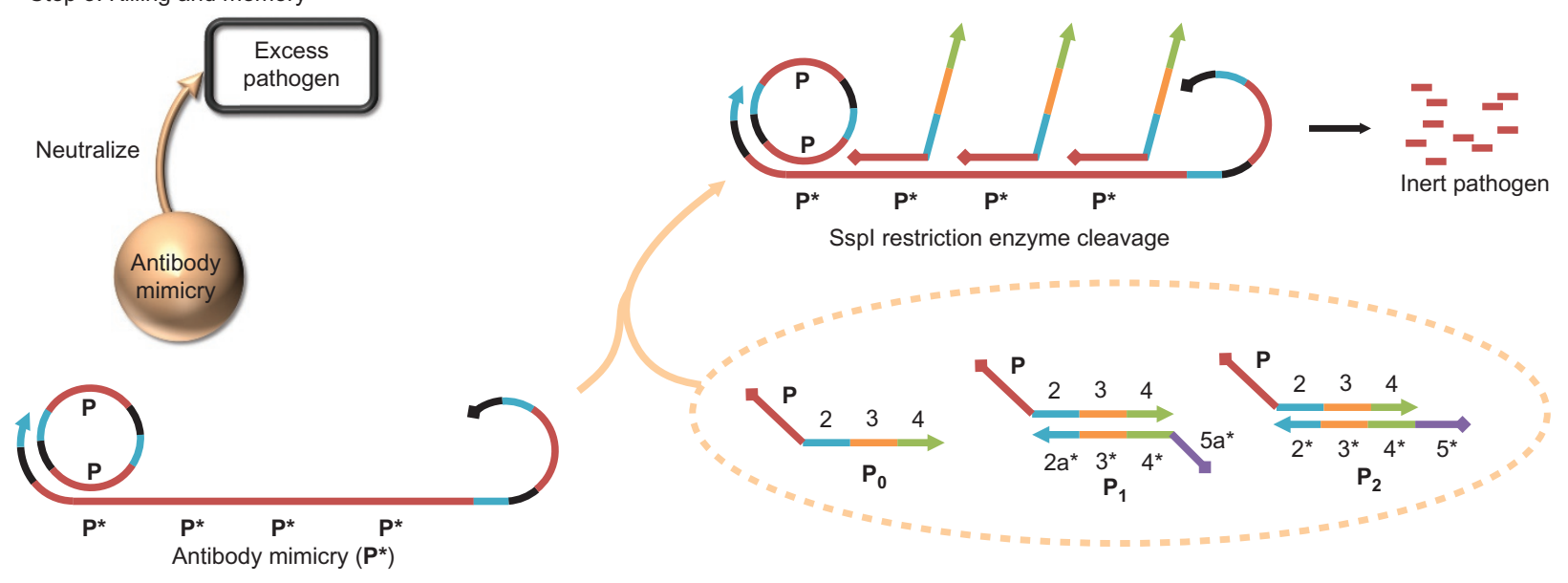

Figure 1 | Working principle of the AIRS. AIRS consists of three steps: (1) recognition and tolerance, (2) immune response and (3) killing and memory. These functions are blocked in the absence of ssDNA pathogen input $\left(\mathbf{P}_{\mathbf{0}}\right)$, but once $\mathbf{P}_{\mathbf{0}}$ is introduced to the AIRS system it is driven by a series of DNA toehold-mediated strand displacement and DNA-enzyme reactions. Coloured bars indicate DNA strands with different domains. All $x$ domains are complementary to $x^{*} ; \mathbf{P}_{\mathbf{0}}$ is the pathogen sequence that possesses infection ability in the ssDNA form. AM (antigen-presenting cell mimicry), BM (B-cell mimicry) and PG (primer generator, which generates primers for the analogue antibody) are initially present as duplex components, along with CT (circular template, which controls the sequence of the analogue antibody) and two functional enzymes, Phi29 polymerase and Sspl restriction enzyme. TM ( $T$ Cell Mimicry); RCA (rolling cycle amplification). $\mathrm{P}_{1}$ and $\mathrm{P}_{2}$ are displacement reaction products.

tolerance, immune response, and killing and memory) under the control of four functional DNA components, including the DNA duplexes AM (antigen-presenting cell (APC) mimicry), BM
(B-cell mimicry), PG (primer generator, which generates primers for the analogue antibody) and single-stranded circular DNA CT (circular template, which controls the sequence of the analogue 
antibody), as well as two enzymes (Phi29 DNA polymerase and SspI restriction enzyme) able to autonomously and programmably respond to an incoming piece of single-stranded DNA (ssDNA) pathogen input $\left(\mathbf{P}_{\mathbf{0}}\right)$ taken from bacterial genome. When no $\mathbf{P}_{\mathbf{0}}$ is present, the system is maintained in a steady, balanced state by the effective blocking of the functional domains in each component. However, when challenged by $\mathbf{P}_{\mathbf{0}}$, these functional domains are activated in a series of steps designed to mimic the three steps given above.

In step 1 , in the absence of $\mathbf{P}_{\mathbf{0}}$, the functional components of the system remain in stasis. Mimicking immune tolerance, the unresponsive state of immune defence, the reaction priority of $\mathbf{P}_{\mathbf{0}}$ to duplex DNA AM, as well as to $\mathbf{B M}$, is controlled through the lengths of their corresponding toeholds, which are short ssDNA overhang segments in duplexes. Accordingly, we designed a ten nucleotide (nt) toehold in AM with a displacement reaction rate $k$ of $10^{6} \mathrm{M}^{-1} \mathrm{~s}^{-1}$ and a $0 \mathrm{nt}$ toehold in $\mathbf{B M}$ with a $k$ value of $10^{3} \mathrm{M}^{-1} \mathrm{~s}^{-1}$ for the initial $\mathbf{P}_{\mathbf{0}}$ hybridization ${ }^{17,18}$. AM can display $\mathbf{P}_{\mathbf{0}}$, which has two ssDNA domains, one having a sequence taken from the genome of Bacillus anthracis, a Gram-positive, rodshaped bacterium (P), and the other designed (ssDNA domain 2-3-4) to control downstream reactions through a DNA-displacement reaction. However, at the recognition and tolerance phase, our system can only proceed to step 2, immune response, when the amount of pathogen accumulated exceeds the threshold of AM.

Once the threshold of immune tolerance is reached in the system, step 2, the analogue humoral immune response, is activated. As previously noted, during the depletion of $\mathbf{A M}$ by the displacement reaction, one of the displaced products, ssDNA TM (T-cell mimicry), accumulates and can be used as a catalyst to increase the reaction rate between $\mathbf{P}_{\mathbf{0}}$ and $\mathbf{B M}$, and thus release a primer (ssDNA domain 12a) for the DNA polymerase-catalysed rolling-circle amplification (RCA), which this system will employ to produce DNA 'antibody' mimicry $\left(\mathbf{P}^{\star}\right)$ to the antigen B. anthracis $(\mathbf{P})$. Thus, to replicate the host-defence immune response, we incorporated two $B$. anthracis genomic sequence fragments $(\mathbf{P})$ in the $\mathbf{C T}$, which resulted in a rapid generation of the complementary sequences $\mathbf{P}^{\star}$, essentially by enzymatic amplification, using Phi29 DNA polymerase, the replicative polymerase from the $B$. subtilis phage phi29, and deoxyribonucleotide triphosphate (dNTP), to mimic the fast and antibody-specific generation naturally produced by B cells.

In step 3, killing and memory, of the proposed system it was necessary to mimic the process by which cell-mediated immune response eliminates pathogens after the formation of an antibodyantigen complex from the body, which, as noted above, results from activation of the humoral immune response by $\mathrm{B}$ lymphocytes. To accomplish this, three infectious states were assumed: original pathogen strand $\mathbf{P}_{\mathbf{0}}$ and the displacement reaction products (with $\mathbf{A M}$ and $\mathbf{B M}) \mathbf{P}_{\mathbf{1}}$ and $\mathbf{P}_{\mathbf{2}}$. Together, these all contain an infectious pathogen domain $(\mathbf{P})$. Then, to form the antibody-antigen complex in the system, the $\mathbf{P}$ domain in $\mathbf{P}_{\mathbf{0}}, \mathbf{P}_{\mathbf{1}}$ and $\mathbf{P}_{\mathbf{2}}$ hybridizes with a large amount of $\mathbf{P}^{\star}$ and forms stable duplex regions $\mathbf{P} \mathbf{P}^{\star}$. However, although the vertebrate host immune system uses phagocytosis as a key strategy in removing pathogens, we designed a purely biomechanical method to mimic the activation of the humoral immune response, that is the binding between the generated $\mathbf{P}^{\star}$ and the $\mathbf{P}$ domain from the pathogen, followed by a restriction enzyme-based step that mimics cell-mediated killing by specifically cutting the 'antibody-antigen' duplex. More specifically, this latter step is accomplished when the hybridization of the $\mathbf{P}$ domain with $\mathbf{P}^{\star}$ creates an active recognition site for the restriction enzyme SspI, which is extracted from an E. coli strain that carries the cloned and modified (Y98F) sspi gene from the restriction site of Sphaerotilus species. In this way, the $\mathbf{P}$ domain in $\mathbf{P}_{\mathbf{0}}, \mathbf{P}_{\mathbf{1}}$ and $\mathbf{P}_{\mathbf{2}}$ can be specifically cut into two fragments with the nucleotide numbers 56 and 15 (ref. 19), thus losing infectivity.
Next, in the adaptive immune system, some $\mathrm{T}$ and $\mathrm{B}$ cells will become memory cells able to 'remember' specific pathogens. These memory cells will trigger a much faster immune response on subsequent exposure to the same pathogen. Therefore, to create a mechanism to mimic the memory function of B cells, we turned to ssDNA TM, which is produced by the displacement reaction between $\mathbf{P}_{\mathbf{0}}$ and $\mathbf{A M}$ and remains in the system from the first exposure to $\mathbf{P}_{\mathbf{0}}$. We also describe its catalysis of $\mathbf{P}_{\mathbf{0}}$ and $\mathbf{B M}$ displacement to release primer quickly, and thus trigger a faster RCA reaction that creates antibody for the next exposure to the same pathogen sequence. This reaction is defined as entropy driven because it is one that spontaneously evolves towards thermodynamic equilibrium (Supplementary Fig. 7) ${ }^{18}$. Without $\mathbf{T M}$, the displacement rate between $\mathbf{P}_{\mathbf{0}}$ and $\mathbf{B M}$ would be slowed by the effective blocking of active domains on $\mathbf{B M}$. However, in the presence of $\mathbf{T M}, \mathbf{B M}$ can hybridize with $\mathbf{T M}$ through its $4 \mathrm{nt}$ toehold (domain $5^{*}$ ) and then release the ssDNA primer initiator $(\mathbf{P I})$ and the side product $\mathrm{W}_{1}$, which results in a new toehold binding region (domain $3^{*}$ ) for the hybridization between $\mathbf{P}_{\mathbf{0}}$ and $\mathbf{B M}$, which is driven forwards thermodynamically by the entropic gain of the liberated molecules. Thus, the effect of memory is produced by speeding up the reaction rate between $\mathbf{P}_{\mathbf{0}}$ and $\mathbf{B M}$ using the catalytic effect of the remaining $\mathbf{T M}$ produced by the initial recognition. Thus, by leaving TM as a 'catalytic fuel' in the system, a memory effect for a specific pathogen input is achieved.

Verification of signal transduction. To ensure the proper operation of the entire network, signal transduction at each step was validated separately. To examine the precision of the threshold function of AM, we used a Förster resonance energy transfer (FRET)-based method to study the hybridization order of $\mathbf{P}_{\mathbf{0}}$ with $\mathbf{A M}$ and $\mathbf{B M}$. A fluorophore (fluorescein (FAM)) and quencher (DABCYL $(\mathrm{DAB})$ ) pair was coupled in the PG duplex to indicate the released amount of primer for the RCA reaction. Fluorescence restoration only occurs if the threshold level of AM is exceeded, after which the reaction proceeds to step 2, immune response, based on strand-displacement reactions that can release the quencher-labelled primer strand. Therefore, in the presence of $80 \mathrm{nM}$ AM, $100 \mathrm{nM} \mathbf{B M}$ and $100 \mathrm{nM}$ fluorophore/quencher-labelled PG, different concentrations of $\mathbf{P}_{\mathbf{0}}$ (from 0 to $150 \mathrm{nM}$ ) were introduced to the system with fluorescence monitoring at $517 \mathrm{~nm}$. As shown in Fig. $2 b$, the fluorescence restoration exhibited a sharp upturn at the $\mathbf{P}_{\mathbf{0}}$ concentration of $80 \mathrm{nM}$, but little increase below $80 \mathrm{nM}$, which indicates that the reaction between $\mathbf{P}_{\mathbf{0}}$ and $\mathbf{B M}$ had begun at the depletion point of $\mathbf{A M}$. In other words, the threshold value of $\mathbf{A M}$ to $\mathbf{P}_{\mathbf{0}}$ is adjustable by changing the concentration of AM. In this case, the depletion point, or threshold level, of $\mathbf{A M}$ was reached at $80 \mathrm{nM}$, indicating that the reaction between $\mathbf{P}_{\mathbf{0}}$ and $\mathbf{B M}$ could initiate step 2, which, in humans, is the production of antibodies by $\mathbf{B}$ lymphocytes. To confirm this result further, the fluorescence kinetics of the above system was monitored. Initially, $80 \mathrm{nM} \mathbf{A M}, 100 \mathrm{nM} \mathbf{B M}$ and $100 \mathbf{n M}$ PG were mixed in the buffer, and a small amount of $\mathbf{P}_{\mathbf{0}}$ (30 $\mathrm{nM}$ ) was added. As a result of the excess amount of $\mathbf{A M}$ and its reaction priority to $\mathbf{P}_{\mathbf{0}}$ by the requirements of step 1 , slow fluorescence restoration kinetics occurred. However, when more $\mathbf{P}_{\mathbf{0}}$ (30 $\mathrm{nM}$ each time) was added continuously to the system, a sharp increase (after $90 \mathrm{nM}$ in total) was seen in the fluorescence kinetics, which indicates that the reaction could proceed to step 2 . Different threshold values were tested by changing the concentrations of $\mathbf{A M}$ and $\mathbf{B M}$ (Supplementary Fig. 1). Gel electrophoresis was also used to indicate the order of reactions (Supplementary Fig. 2). As the same recognition domain (2-3-4) is shared, similar experimental results were also observed by substituting $\mathbf{P}_{\mathbf{0}}$ for another pathogen sequence, $\mathbf{P}_{\mathbf{0}}$-SARS (severe acute respiratory syndrome coronavirus genomic DNA) (Supplementary Fig. 3). All the assays confirmed the effective 

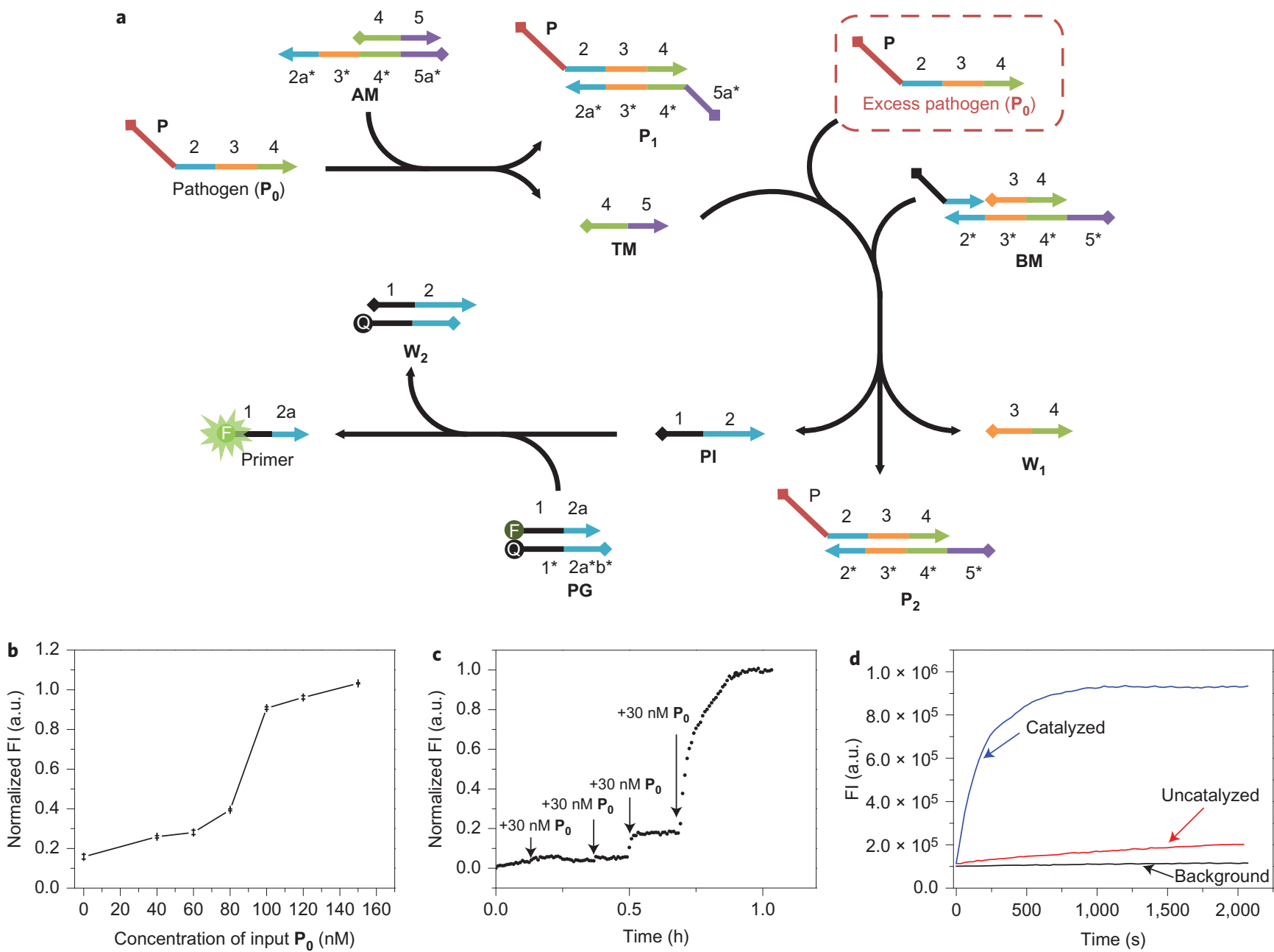

Figure $\mathbf{2}$ | Scheme and results that confirm the reaction priority between steps 1 (recognition and tolerance) and $\mathbf{2}$ (immune response). a, Scheme of reaction steps that occur in steps 1 and 2 . $\mathbf{W}_{\mathbf{2}}$ is the side product from the displacement reaction of $\mathbf{P I}$ and $\mathbf{P G}$. $\mathbf{b}$, Plot of fluorescence restoration of the system with 80 nM AM, 100 nM BM and 100 nM FAM/DAB-labelled PG with different concentrations of the ssDNA pathogen $\mathbf{P}_{\mathbf{0}}$. $\mathbf{c}$, Kinetics experiments of the system with $80 \mathrm{nM}$ AM, 100 nM BM and 100 nM FAM/DAB-labelled PG. At each time point, $30 \mathrm{nM} \mathbf{P}_{\mathbf{o}}$ were added to the buffer separately. d, Kinetics experiments to study the catalytic effect of TM. The black curve represents the background kinetics with $80 \mathrm{nM}$ AM, $100 \mathrm{nM}$ BM, $100 \mathrm{nM}$ FAM/DAB-labelled $\mathbf{P G}$ and no $\mathbf{P}_{\mathbf{0}}$. The red curve shows the uncatalysed reaction kinetics with $100 \mathrm{nM} \mathbf{B M}, 100 \mathrm{nM}$ FAM/DAB-labelled PG and 150 nM $\mathbf{P}_{\mathbf{0}}$. The blue curve exhibits the catalysed reaction kinetics with $80 \mathrm{nM}$ AM, $100 \mathrm{nM}$ BM, $100 \mathrm{nM}$ FAM/DAB-labelled PG and $150 \mathrm{nM} \mathbf{P}_{\mathbf{0}}$. The experiments were performed at $25^{\circ} \mathrm{C}$ in $50 \mathrm{mM}$ Tris-HCl buffer that contained $10 \mathrm{mM} \mathrm{MgCl}$. a.u., arbitrary units.

threshold function of $\mathbf{A M}$ relative to the reaction between $\mathbf{B M}$ and $\mathbf{P}_{\mathbf{0}}$, and thus provided a macroscopic mimicry of the 'recognition and tolerance' state of AIS.

Another significant function of $\mathbf{A M}$ is to produce catalytic ssDNA TM to facilitate the hybridization reaction between $\mathbf{P}_{\mathbf{0}}$ and BM, which, according to Zhang et al. ${ }^{18}$, can accelerate the hybridization reaction by two to four orders of magnitude. ssDNA TM is not only important in facilitating the hybridization between $\mathbf{P}_{\mathbf{0}}$ and $\mathbf{B M}$, but it is also key to the formation of 'mimetic memory cells'. Therefore, to prove the catalytic effect of TM, we continued to use our FRET-based reporting model, as described above. Accordingly, $80 \mathrm{nM} \mathbf{A M}, 100 \mathrm{nM} \mathbf{B M}$ and $100 \mathrm{nM}$ FAM/DAB-labelled PG were incubated in buffer. Afterwards, $150 \mathrm{nM} \mathbf{P}_{\mathbf{0}}$ were added to the system. We used an excess amount of $\mathbf{P}_{\mathbf{0}}$ to $\mathbf{A M}$ to overcome the threshold value. TM is generated in step 1 as a result of the reaction between $\mathbf{P}_{\mathbf{0}}$ and $\mathbf{A M}$. Thus, $\mathbf{P}_{\mathbf{0}}$, when it exceeds the threshold (as set by controlling the concentration of $\mathbf{A M}$ ), in turn results in a catalytic reaction between excess $\mathbf{P}_{\mathbf{0}}$ and $\mathbf{T M}$ by entropy that can quickly restore the fluorescence of the system. As a comparison, the same concentration of $\mathbf{P}_{\mathbf{0}}$ was added directly to a solution with $100 \mathrm{nM}$ BM and 100 nM FAM/DAB-labelled PG, but no AM. As shown in Fig. 2d, measurements of the fluorescence kinetics of the catalysed reaction exhibited an over 500 -fold acceleration in contrast to that without catalysis ${ }^{18,20}$. Thus, as explained above, the released TM could serve as specific memory mimicry, which results in a stronger and faster immune response for the next exposure to the same pathogen. Afterwards, we also confirmed that the quantity of $\mathbf{P}^{*}$ generated from enzymatic amplification for hybridization with domain $\mathbf{P}$ in $\mathbf{P}_{\mathbf{0}}, \mathbf{P}_{\mathbf{1}}$ and $\mathbf{P}_{\mathbf{2}}$ could be determined by the amount of primer, thus making the enzymatic reaction controllable by upstream reactions in steps 1 and 2 (see the Supplementary Information). In addition, the restriction-enzyme-based digestion of the generated antigen-antibody complex was studied and is shown in the Supplementary Information.

Whole-system performance. After confirming the proper function of each step, we further tested the performance of the entire system with the same pathogen strand input from the B. anthracis genome $\left(\mathbf{P}_{\mathbf{0}}\right)$. To confirm the tolerance of AIRS, a small amount of $\mathbf{P}_{\mathbf{0}}$ $(100 \mathrm{nM})$ was then added to the system, and fluorescence was monitored in real time. Next, we designed a DNA molecular beacon (MB-R) to report the amount of RCA product fluorescently by 
a

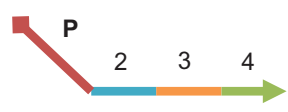

Pathogen $\left(\mathbf{P}_{\mathbf{0}}\right)$
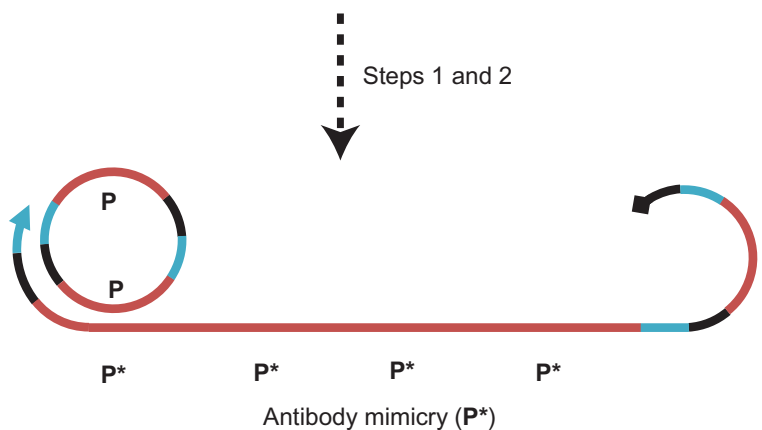

$4^{M B-R}$

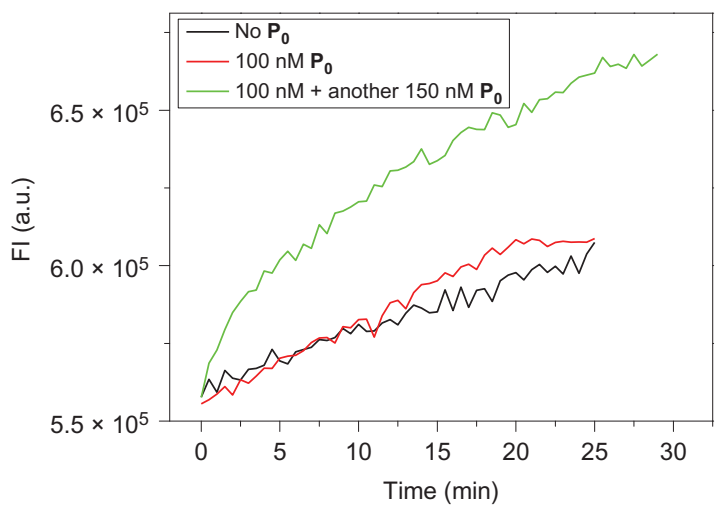

b

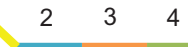

Pathogen ( $\mathbf{P}_{0}$-SARS)
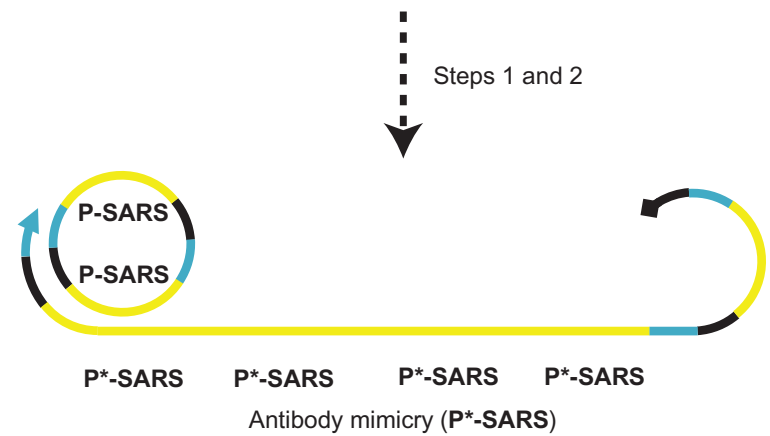

MB-R-SARS

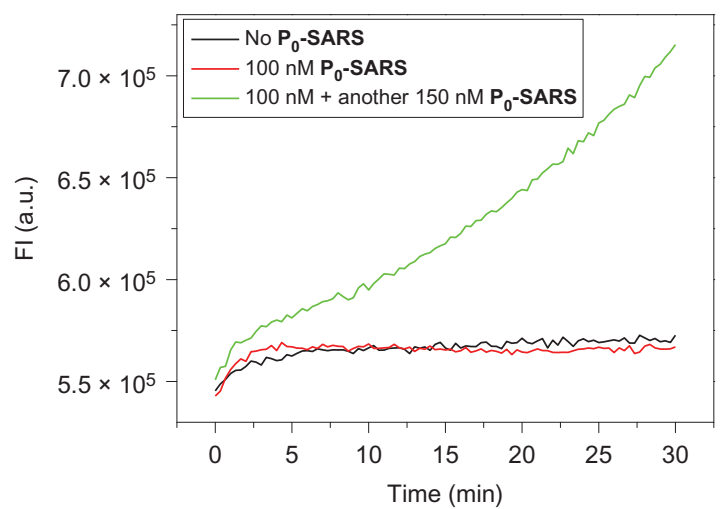

Figure 3 | Validation of signal transduction in the entire AIRS mimicry network by fluorescence. $\mathbf{a}, \mathbf{b}$, The fluorescence kinetics of the antibody mimicry $\mathbf{P}^{\star}\left(\mathbf{P}^{\star}\right.$-SARS) generation was monitored with different concentrations of $\mathbf{P}_{\mathbf{0}}(\mathbf{a})$ and $\mathbf{P}_{\mathbf{0}}$-SARS (b). Here we used double the amounts of the individual DNA components compared with those used for the data shown in Fig. 2 to ensure that the concentrations were consistent with the gel experiment.

using the pathogen B. anthracis genomic sequence $\mathbf{P}$ as its loop. MB-R could be opened by the RCA products, that is $\mathbf{P}^{\star}$, and thereby exhibit fluorescence restoration that represents different amounts of $\mathbf{P}^{\star}$. Figure $3 \mathrm{a}$ shows that the fluorescence kinetics is similar to that without $\mathbf{P}_{\mathbf{0}}$, which confirms that the system is not activated to produce $\mathbf{P}^{\star}$ when the amount of pathogen is below the level of immune tolerance (that is, a threshold of $200 \mathrm{nM}$ ). However, when another $150 \mathrm{nM} \mathbf{P}_{\mathbf{0}}$ was added to the system to mimic a second exposure to the same pathogen, the fluorescence restoration showed a much faster kinetics behaviour, which indicates the production of a large amount of $\mathbf{P}^{\star}$ and a successful triggering of the immune response. In addition, the faster response during the second exposure also confirms the memory effect of this system for a specific pathogen. If $\mathbf{P}_{\mathbf{0}}$-SARS is used as a pathogen input and a molecular beacon encoded with $\mathbf{P}_{\mathbf{0}^{-}}$-SARS (MB-R-SARS) is used as the reporter, similar fluorescence-response trends are seen, as shown in Fig. 3b, which indicates that AIRS potentially works for a second pathogen input. We also used a different fluorescence-reporting system to indicate the amount of generated $\mathbf{P}^{\star}$, which is shown in Supplementary Fig. 10.

Using a fluorescence reporter in independent experiments, AIRS was demonstrated to operate equally well with two different pathogen inputs. Therefore, we next studied if it could also work when two different inputs were presented simultaneously. To make this determination, $\mathbf{P}_{\mathbf{0}}$ and $\mathbf{P}_{\mathbf{0}}$-SARS were introduced together to a solution that contained all the components $(300 \mathrm{nM}$ AM, $300 \mathrm{nM}$

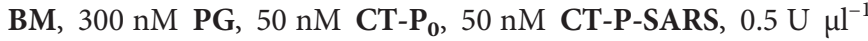
Phi29 and $250 \mu \mathrm{M}$ dNTP). As in the experiment above, two different molecular beacons with the same fluorophore and quencher (MB-R and MB-R-SARS) were also added to the mixture to report the amounts of both RCA products fluorescently, that is $\mathbf{P}^{\star}$ and $\mathbf{P}^{\star}$-SARS. As shown in Supplementary Fig. 14, as long as both pathogen inputs are below the level of immune tolerance (threshold of $300 \mathrm{nM}$ ), AIRS is not activated and fluorescence remains unchanged compared with the background signal. However, when the concentrations increase to a level higher than the threshold for either one, or both, pathogens, fluorescence is restored, which indicates that both pathogen inputs can generate corresponding antibody mimicry when they are mixed together. This confirms that AIRS has the capacity to respond simultaneously to two different pathogen inputs.

To characterize further the error-free operation of the AIRS system, we used gel electrophoresis to analyse the final amount of $\mathbf{P}^{\star}$. As shown in Fig. 4a, only one sharp band of high molecular weight can be seen when the amount of pathogen exceeds the threshold level $(100+150 \mathrm{nM})$, which is consistent with the previous fluorescence results. To study the binding between the pathogen input strand $\left(\mathbf{P}_{\mathbf{0}}\right)$ and the antibody mimicry strand $\left(\mathbf{P}^{\star}\right)$ in step 3 , we modified $\mathbf{P}_{\mathbf{0}}$ with a fluorophore (FAM). After the generation of $\mathbf{P}^{\star}$, the hybridization between $\mathbf{P}_{\mathbf{0}}$ and $\mathbf{P}^{\star}$ was confirmed to be present, as a band of high molecular weight could be seen in the fluorescein isothiocyanate (FITC)-imaging channel 
a

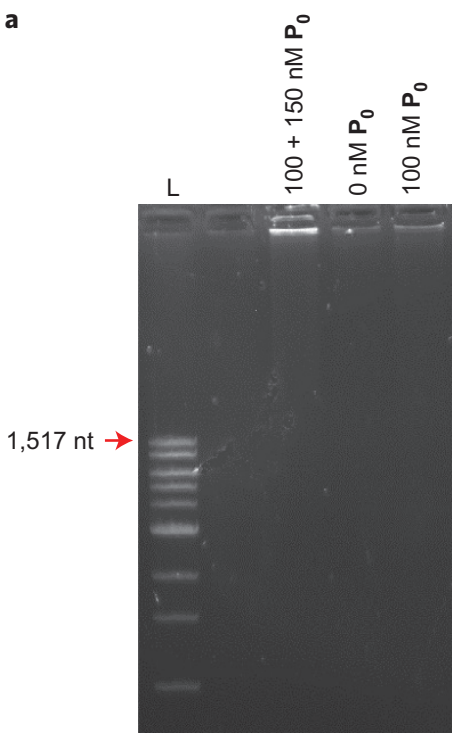

b

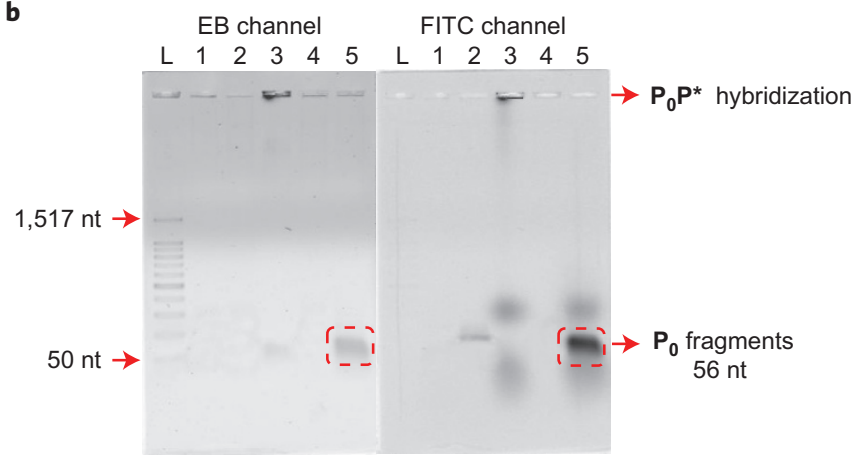

C $\mathrm{L} 1 \quad \begin{array}{llllllllllll} & 2 & 3 & 4 & 5 & \mathrm{~L} & 1 & 2 & 3 & 4 & 5\end{array}$

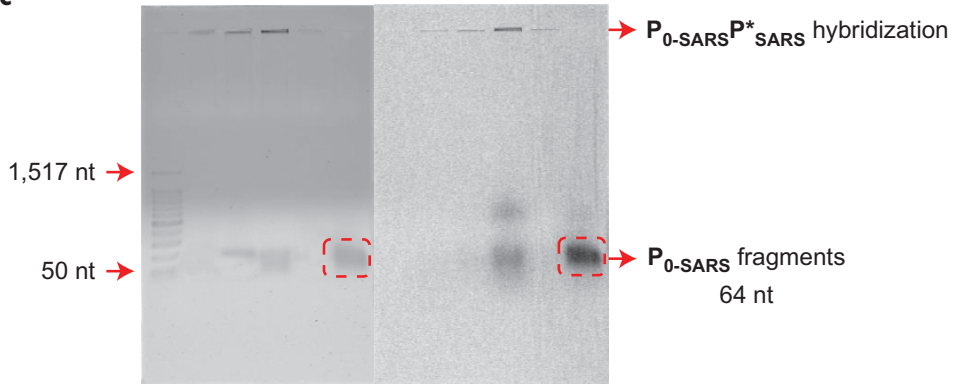

Figure 4 | Characterization of error-free operations of the entire AIRS system by gel electrophoresis. $\mathbf{a}$, Analysis of antibody mimicry $\mathbf{P} \star$ generation by agarose gel (1.5\%). L, 100-base-pair ladder. The image was taken under an ethidium bromide $($ EB $)$ imaging channel $\left(\lambda_{\text {excitation }}=520 \mathrm{~nm}, \lambda_{\text {emission }}=605 \mathrm{~nm}\right)$. b, Validation of the AIRS analogue system performance with pathogen strand $\mathbf{P}_{\mathbf{0}}$ by agarose gel (1\%). $\mathrm{M}$ is the mixture of original present components in the AIRS. Here M = mixture of 200 nM AM, 200 nM BM, 200 nM PG, 50 nM CT, $0.5 U_{\mu l}^{-1}$ Phi29 and $250 \mu M$ dNTP; incubation time, one hour. Lane 1, M + 0 nM FAM-labelled $\mathbf{P}_{\mathbf{0}}$; lane 2, M + 100 nM FAM-labelled $\mathbf{P}_{\mathbf{0}}$; lane 3, M + 500 nM FAM-labelled $\mathbf{P}_{\mathbf{0}}$; lane 4, M + 100 nM FAM-labelled $\mathbf{P}_{\mathbf{0}}+2$ U $\mu l^{-1}$ Sspl; lane 5 , $\mathrm{M}+500 \mathrm{nM}$ FAM-labelled $\mathbf{P}_{\mathrm{O}}+2 \mathrm{U} \mathrm{\mu l}^{-1} \mathrm{Sspl}$; $\mathrm{L}, 1 \mathrm{~kb}$ ladder. $\mathbf{c}$, Validation of the AIRS analogue system performance with the pathogen strand $\mathbf{P}_{0}$-SARS by agarose gel (1\%). M = mixture of 200 nM AM, 200 nM BM, 200 nM PG, 50 nM CT-SARS, $0.5 \cup \mu{ }^{-1}$ Phi29 and $250 \mu M$ dNTP; incubation time, one hour. Lane 1, M + 0 nM FAM-labelled $\mathbf{P}_{0}$-SARS; lane 2, M + 100 nM FAM-labelled $\mathbf{P}_{0}$-SARS; lane 3, M + 500 nM FAM-labelled $\mathbf{P}_{0}$-SARS; lane 4, M + 100 nM

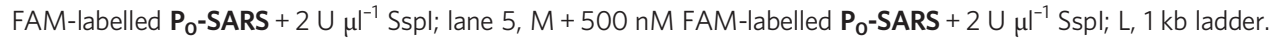

(Supplementary Fig. 13). With the addition of more FAM-labelled $\mathbf{P}_{\mathbf{0}}$ (from one to 250 excess) to the system, the hybridization between excess $\mathbf{P}_{\mathbf{0}}$ and generated $\mathbf{P}^{\star}$ was still observed, which indicates that AIRS could sufficiently bind the excess pathogen input strand $\left(\mathbf{P}_{\mathbf{0}}\right)$ by RCA. Finally, we incubated the restriction enzyme $\left(2 \mathrm{U} \mathrm{Hl}^{-1}\right)$ together with all other components (200 nM AM, $200 \mathrm{nM}$

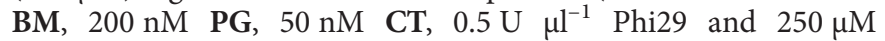
dNTP). After introducing an excess concentration of FAM-labelled $\mathbf{P}_{\mathbf{0}}(500 \mathrm{nM})$ to surpass the immune tolerance of the system, a digestion fragment band with a FAM modification $(56 \mathrm{nt})$ was discovered under the FITC channel, which leads to the conclusion that the pathogen had been successfully (Fig. 4b) digested. Interestingly, if $\mathbf{P}_{\mathbf{0}}$ is substituted by a double-nucleotide polymorphism input $\left(\mathbf{P}_{0}\right.$-mis), antibody mimicry $\mathbf{P}^{\star}$ can still be generated with an excess concentration of $\mathbf{P}_{0^{-}}$-mis. However, the hybridization between $\mathbf{P}_{\mathbf{0}}$-mis and $\mathbf{P}^{\star}$ cannot be digested by the SspI restriction enzyme, which demonstrates that the AIRS system is sensitive to the recognition of nucleotide polymorphism of the pathogen input (Supplementary Fig. 11). AIRS has been designed with two layers of selectivity, which makes it possible for the system to differentiate $\mathbf{P}_{\mathbf{0}}$ from a double-nucleotide polymorphism input, $\mathbf{P}_{\mathbf{0}}$-mis. To explain, the relatively weaker binding ability between $\mathbf{P}_{\mathbf{0}}$-mis and $\mathbf{P}^{\star}$ when compared to that between $\mathbf{P}_{\mathbf{0}}$ and $\mathbf{P}^{\star}$ makes the $\mathbf{P}_{\mathbf{0}}$-mis- $\mathbf{P}^{\star}$ duplex less stable. Additionally, a $\mathbf{P}_{\mathbf{0}}$-mis- $\mathbf{P}^{\star}$ mismatch duplex cannot form an effective recognition binding site with the restriction enzyme SspI (AAT-ATT), and thereby reduce the enzyme's digestion rate, which, in turn, results in much less cleavage product from the input $\mathbf{P}_{\mathbf{0}^{-}}$-mis (Supplementary Fig. 11). In contrast, because the hybridization of $\mathbf{P}_{\mathbf{0}}$-SARS with $\mathbf{P}^{\star}$ creates a recognition binding site identical to that of the restriction enzyme SspI, similar gel electrophoresis results were found (Fig. 4c), which again confirms that AIRS can work for different pathogen inputs.

Finally, as noted above, the specificity of the system mainly depends on the restriction enzyme and sequence of CT (see the Supplementary Information). When we challenged the system by introducing FAM-labelled $\mathbf{P}_{\mathbf{0}}$-SARS as the pathogenic input, but CT for B. anthracis as the amplification template, no obvious fluorescent band of high molecular weight appeared with either small or large amounts of $\mathbf{P}_{\mathbf{0}}$-SARS under the FITC channel because of the lack of hybridization between $\mathbf{P}_{\mathbf{0}}$-SARS and $\mathbf{P}^{\star}$ specifically generated for B. anthracis (Supplementary Fig. 12).

\section{Conclusions}

We began by hypothesizing the feasibility of designing a network of chemical reactions able to function in a manner similar to the vertebrate AIS, using DNA-DNA and DNA-enzyme interactions as simplified artificial analogues to mimic the macroscopic behaviours of the vertebrate adaptive immune system. Accordingly, the designed artificial AIRS was, de novo, demonstrated to mimic superficially three basic steps of the host immune response, namely recognition and tolerance, immune response, and killing and memory, but without the presence any living component to achieve functionality. Moreover, even though the designed system is entirely synthetic, biocompatibility was shown by its ability to use only biological molecules (DNA and enzyme). Consequently, as the cellular state can be controlled by DNA or RNA, it could be possible to encode cellular functions to the system output (that is, the DNA and/or RNA strand) $)^{5,21-26}$, which makes it possible to scale up this prototype to increasingly intelligent molecular nanocontrollers and nano-operators. However, to achieve this, our 
current construction design will need to be improved and integrated with other chemistries.

\section{Methods}

Preparation of DNA CT. A $7.2 \mu \mathrm{l}$ sample of $20 \mu \mathrm{M}$ CT (with a phosphate group on its $5^{\prime}$ end) was placed in $4 \mu \mathrm{l}$ CircLigase II $10 \mathrm{x}$ reaction buffer that contained $330 \mathrm{mM}$ Tris-acetate (pH 7.5), $0.66 \mathrm{M} \mathrm{KAc}, 5 \mathrm{mM}$ dithiothreitol (DTT) and $2.5 \mathrm{mM}$ $\mathrm{MnCl}_{2}$. Afterwards, $2.8 \mu \mathrm{l}$ CircLigase II (Epicentre) was mixed with the buffer and diluted to $40 \mu \mathrm{l}$ with water. The mixture was incubated at $60^{\circ} \mathrm{C}$ for three hours. Then $20 \mathrm{U}$ Exonuclease I and $200 \mathrm{U}$ Exonuclease III (New England Biotech) were added to the mixture and incubated at $37^{\circ} \mathrm{C}$ for one hour. The enzyme was denatured by heating the solution to $90^{\circ} \mathrm{C}$ for 20 minutes. Finally, the CT product was purified by denatured PAGE and desalted with NAP-5 columns.

Validation of signal transduction in each step and the entire system by fluorescence. When the reaction priority between step 1 and step 2 was tested, purified AM (50 nM), BM (50 nM) and PG (with FAM and DAB, $50 \mathrm{nM}$ ) were mixed in $1 \times$ TAE (Tris base, acetic acid and EDTA) $-\mathrm{Mg}$ buffer to a total volume of $100 \mu \mathrm{l}$ and the fluorescence was monitored for different concentrations of $\mathbf{P}_{\mathbf{0}}$ (or $\mathbf{P}_{\mathbf{0}}$-SARS or $\mathbf{P}_{\mathbf{0}^{0}}$-mis) (Supplementary Fig. 1). Fluorescence intensities (FIs) were tested after incubation for one hour at room temperature. When the signal transduction in the entire system was tested by fluorescence, purified AM (200 nM), BM (200 nM), PG (no FAM or DAB, 200 nM), 50 nM CT (or CT-SARS) and 500 nM MB-R (or 200 nM DNA hairpin $\mathbf{A}_{1}, 200$ nM DNA hairpin $\mathbf{A}_{2}$ and $250 \mathrm{nM}$ fluorescent reporter $\mathbf{R}_{\mathbf{1 2}}$, (for another fluorescence-reporting method described in Supplementary Fig. 9) were incubated in $1 \times \mathrm{TAE}-\mathrm{Mg}^{2+}$ buffer $(50 \mathrm{mM}$ Tris- $\mathrm{HCl}$, $10 \mathrm{mM} \mathrm{MgCl}_{2}$ ). The fluorescence kinetics was started when different amounts of $\mathbf{P}_{\mathbf{0}}$ were added to the solution. To check the fluorescence kinetics of the system on a second exposure to $\mathbf{P}_{\mathbf{0}}, 100 \mathrm{nM} \mathbf{P}_{\mathbf{0}}$ were first added to a buffer that contained purified AM (200 nM), BM (200 nM), PG (no FAM or DAB, $200 \mathrm{nM}$ ) and $50 \mathrm{nM}$

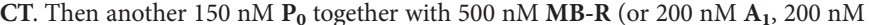
$\mathbf{A}_{\mathbf{2}}$ and $250 \mathrm{nM} \mathrm{\mathbf {R } _ { \mathbf { 1 2 } }}$ ) were mixed together in the solution simultaneously.

Electrophoresis analysis of the system by agarose gel. A $10 \mu \mathrm{l}$ system with purified

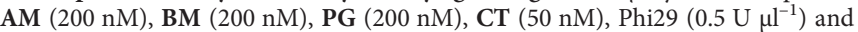
dNTP $(250 \mu \mathrm{M})$ was chosen for this experiment. For the pathogen-digestion

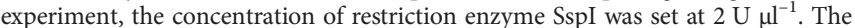
reaction was carried out in $1 \times \mathrm{NEB}$ buffer $\left(50 \mathrm{mM}\right.$ Tris- $\mathrm{HCl}, 10 \mathrm{mM} \mathrm{MgCl}{ }_{2}$, $10 \mathrm{mM}\left(\mathrm{NH}_{4}\right)_{2} \mathrm{SO}_{4}$ and $\left.4 \mathrm{mM} \mathrm{DTT}\right)$ with different amounts of $\mathbf{P}_{\mathbf{0}}$ input at $30^{\circ} \mathrm{C}$ for three hours. The agarose gel was run at $4{ }^{\circ} \mathrm{C}$ for one hour under $100 \mathrm{~V}$.

Received 5 December 2014; accepted 10 July 2015; published online 17 August 2015

\section{References}

1. Bottomly, K. 1984: all idiotypes are equal, but some are more equal than others. Immunol. Rev. 79, 45-61 (1984).

2. Krishnan, Y. \& Simmel, F. C. Nucleic acid based molecular devices. Angew Chem. Int. Ed. 50, 3124-3156 (2011).

3. Simmel, F. C. Programming the dynamics of biochemical reaction networks. ACS Nano 7, 6-10 (2013).

4. Qian, L. \& Winfree, E. Scaling up digital circuit computation with DNA strand displacement cascades. Science 332, 1196-1201 (2011).

5. Han, D. et al. A logical molecular circuit for programmable and autonomous regulation of protein activity using DNA aptamer-protein interactions. J. Am. Chem. Soc. 134, 20797-20804 (2012).

6. Johne, R., Muller, H., Rector, A., van Ranst, M. \& Stevens, H. Rolling-circle amplification of viral DNA genomes using Phi29 polymerase. Trends Microbiol. 17, 205-211 (2009).

7. Franco, E. et al. Timing molecular motion and production with a synthetic transcriptional clock. Proc. Natl Acad. Sci. USA 108, E784-E793 (2011).

8. Hooshangi, S., Thiberge, S. \& Weiss, R. Ultrasensitivity and noise propagation in a synthetic transcriptional cascade. Proc. Natl Acad. Sci. USA 102, 3581-3586 (2005).
9. Lou, C. et al. Synthesizing a novel genetic sequential logic circuit: a push-on push-off switch. Mol. Syst. Biol. 6, 350 (2010).

10. Padirac, A., Fujii, T. \& Rondelez, Y. Bottom-up construction of in vitro switchable memories. Proc. Natl Acad. Sci. USA 109, E3212-E3220 (2012).

11. Kim, J., White, K. S. \& Winfree, E. Construction of an in vitro bistable circuit from synthetic transcriptional switches. Mol. Syst. Biol. 2, 68 (2006).

12. Qian, L., Winfree, E. \& Bruck, J. Neural network computation with DNA strand displacement cascades. Nature 475, 368-372 (2011).

13. Fujii, T. \& Rondelez, Y. Predator-prey molecular ecosystems. ACS Nano 7, 27-34 (2013).

14. Padirac, A., Fujii, T., Estevez-Torres, A. \& Rondelez, Y. Spatial waves in synthetic biochemical networks. J. Am. Chem. Soc. 135, 14586-14592 (2013).

15. Zhang, D. Y. \& Seelig, G. Dynamic DNA nanotechnology using stranddisplacement reactions. Nature Chem. 3, 103-113 (2011).

16. Zhao, W. A., Ali, M. M., Brook, M. A. \& Li, Y. F. Rolling circle amplification: applications in nanotechnology and biodetection with functional nucleic acids. Angew. Chem. Int. Ed. 47, 6330-6337 (2008).

17. Zhang, D. Y. \& Winfree, E. Control of DNA strand displacement kinetics using toehold exchange. J. Am. Chem. Soc. 131, 17303-17314 (2009).

18. Zhang, D. Y., Turberfield, A. J., Yurke, B. \& Winfree, E. Engineering entropy-driven reactions and networks catalyzed by DNA. Science 318, 1121-1125 (2007).

19. McManus, S. A. \& Li, Y. F. Turning a kinase deoxyribozyme into a sensor. J. Am. Chem. Soc. 135, 7181-7186 (2013).

20. Chen, X. \& Ellington, A. D. Shaping up nucleic acid computation. Curr. Opin. Biotech. 21, 392-400 (2010).

21. Montagne, K., Plasson, R., Sakai, Y., Fujii, T. \& Rondelez, Y. Programming an in vitro DNA oscillator using a molecular networking strategy. Mol. Syst. Biol. 7, 466 (2011).

22. Soengas, M. S., Gutierrez, C. \& Salas, M. Helix-destabilizing activity of phi 29 single-stranded DNA binding protein: effect on the elongation rate during strand displacement DNA replication. J. Mol. Biol. 253, 517-529 (1995).

23. Rothemund, P. W. Folding DNA to create nanoscale shapes and patterns. Nature 440, 297-302 (2006).

24. Hood, L., Heath, J. R., Phelps, M. E. \& Lin, B. Y. Systems biology and new technologies enable predictive and preventative medicine. Science 306, 640-643 (2004).

25. Simmel, F. C. Towards biomedical applications for nucleic acid nanodevices Nanomedicine 2, 817-830 (2007).

26. Xie, Z., Wroblewska, L., Prochazka, L., Weiss, R. \& Benenson, Y. Multi-input RNAi-based logic circuit for identification of specific cancer cells. Science 333, 1307-1311 (2011).

\section{Acknowledgements}

We thank C. Yang, X. Fang, S. Benner, J. Jiang, X. Xiong and K. Williams for discussion and manuscript review. This work is supported by the National Key Scientific Program of China (2011CB911000), by National Natural Science Foundation of China grants (NSFC 21221003 and NSFC 21327009), by the China National Instrumentation Program 2011YQ03012412 and by the National Institutes of Health (GM079359 and CA133086).

\section{Author contributions}

D.H., C.W. and M.Y. contributed equally to this work. D.H. and W.T. conceived the idea and designed the experiment. D.H., C.W., M.Y. and T.Z. performed the experiment. M.Y., T.Z., T.C., C.W., L.Q., S.W., H.L. and Z.Z. provided experimental advice, performed some experiments and analysed the data. D.H., M.Y. and W.T. analysed the data and wrote the manuscript. W.T. supervised the project.

\section{Additional information}

Supplementary information is available in the online version of the paper. Reprints and permissions information is available online at www.nature.com/reprints. Correspondence and requests for materials should be addressed to W.T.

\section{Competing financial interests}

The authors declare no competing financial interests. 